\title{
Pulmonary Delivery: Innovative Approaches and Perspectives
}

\author{
Carlotta Marianecci ${ }^{1}$, Luisa Di Marzio ${ }^{2}$, Federica Rinaldi ${ }^{1}$, Maria Carafa ${ }^{1 *}$, Franco Alhaique $^{1}$ \\ ${ }^{1}$ Department of Drug Chemistry and Technologies, "Sapienza”, University of Rome, Rome, Italy; ${ }^{2}$ Department of Drug Science, \\ University of Chieti “G. D'Annunzio", Chieti, Italy. \\ E-mail: " maria.carafa@uniroma1.it
}

Received October $6^{\text {th }}, 2011$; revised November $17^{\text {th }}, 2011$; accepted November $28^{\text {th }}, 2011$.

\begin{abstract}
The respiratory system, as well as the skin, are organs in direct contact with the environment and they represent possible doors for the entrance of therapeutic agents into the body. Because of the increasing incidence of pulmonary diseases with high mortality and morbidity, pulmonary drug delivery is emerging as a non-invasive and attractive approach for the treatment of several pathologies. It must be pointed out that the development of drug delivery systems for pulmonary application requires a detailed knowledge of the lung, both in its healthy and disease state. Among the various drug delivery systems considered for pulmonary application, nanocarriers show several advantages over other conventional approaches for the treatment of respiratory diseases, for example prolonged drug release and cell-specific targeted drug delivery. Nano-size drug carriers can incorporate various therapeutics (e.g., poorly water soluble drugs, macromolecules) and show interesting features as drug delivery systems to the lung, such as: controlled release, protection from metabolism and degradation, decreased drug toxicity and targeting capabilities. Since gene therapy (e.g. small interfering $R N A$, siRNA) is currently being developed for a wide range of acute and chronic lung diseases, including $C F$, cancer and asthma, the use of nanocarriers for lung release/targeting represents a promising application of such nano-sized structures. Despite the many promising proof of concepts of various delivery technologies reported in this review, further efforts are needed to ensure the safety of long-term in vivo applications and the development of scale up from laboratory to industry in order to reach, together with safety, large-scale production at affordable costs of innovative lung delivery technologies.
\end{abstract}

Keywords: Pulmonary Delivery, Nanovectors

\section{Introduction}

The respiratory system, together with the skin, is an organ in direct contact with the environment and it represents a possible door for the entrance of therapeutic compounds into the body. Because of the increasing incidence of pulmonary diseases with high mortality and morbidity [1], pulmonary drug delivery is emerging as a non-invasive and attractive approach for the treatment of various pathogenic disorders. Such route of administration is acquiring an ever increasing interest also for systemic administration of therapeutic agents. Potential advantages of this route over others, such as the intravenous and the oral ones include:

- rapid drug deposition in the target organ, avoiding high-dose exposures to the systemic circulation;

- rapid onset of drug action;

- lower systemic exposure and consequently reduced side effects;

- evasion of first pass metabolism, because the drugmetabolizing enzymes are in much lower concentrations in the lungs than the gastrointestinal system and liver [2].

In addition, the increasing interest in pulmonary drug delivery is also attributed to the possibility to enhance the bioavailability of molecules with high molecular weight, in comparison with all the other non-injection routes of delivery (oral, buccal, transdermal and nasal), which were shown to be not capable to allow the permeation of macromolecules unless absorption enhancers were used. Furthermore, it must be pointed out that the penetration enhancers, like surfactants and bile salts, may cause significant non-reversible tissue damages, although they increase the permeability of drugs through the epithelial membrane $[3,4]$. Several therapeutic products are investigated for pulmonary delivery with the aim of obtaining 
systemic or local activity. Administration of drugs directly to the lungs is the most appropriate route in the treatment of asthma and other pulmonary diseases such as tuberculosis, chronic obstructive pulmonary disease and lung cancer.

In case of low molecular weight drugs, numerous studies were focused on local application for the treatment of chronic respiratory diseases such as asthma and chronic obstructive pulmonary disease (COPD). At the same time, several recent studies indicated how pulmonary delivery may offer great potential for large molecules, such as proteins and peptides, for both local targeting and systemic effect; i.e., for the treatment of respiratory diseases and other pathologies such as thrombosis and diabetes mellitus. In this sense, inhalation can represent the most favourable non-invasive route of administration for insulin $(5.8 \mathrm{kDa})$ because insulin bioavailability can reach $37 \%$ following inhalation while it reaches at most $1 \%$ following oral, sublingual, nasal or transdermal administration without chemical enhancers $[5,6]$. The first inhaled insulin product, Exubera ${ }^{\circledR}$, was approved in January 2006 but it was withdrawn from the market already in October 2007 due to disappointing sales.

Another inhaled insulin product, AFREZZATM, is currently under review by the FDA for the treatment of type 1 and type 2 diabetes [7,8]. AFREZZA ${ }^{\mathrm{TM}}$ is an ultra rapid acting insulin comprising Technosphere ${ }^{\circledR}$ insulin powder in unit dose cartridges for administration with the inhaler. The Technosphere ${ }^{\circledR}$ powder formulation is prepared by precipitating insulin from a solution onto preformed diketopiperazine particles, which readily dissolve once in the lung environment. AFREZZA ${ }^{\mathrm{TM}}$ appears to overcome several limitations of Exubera ${ }^{\circledR}$. Technosphere ${ }^{\circledR}$ insulin is both rapidly absorbed and eliminated and its pharmacokinetic profile mimics more closely normal physiologic insulin release than injection of regular insulin as well as of rapid-acting analogues

Gene delivery to the lungs is mainly focused on the localized delivery of drugs to the site of action, the lungs and airways, including lung cancer, genetic disorders affecting the airways (cystic fibrosis, alpha-1-antitrypsin deficiency), obstructive lung diseases (asthma), and vaccination. Only one inhaled therapeutic protein is currently available on the market. It is recombinant human deoxyribonuclease I (Dornase alfa) indicated for the treatment of cystic fibrosis (CF) and marketed since 1994.

Recombinant human deoxyribonuclease I is a glycoprotein of $37 \mathrm{kDa}$, which selectively cleaves DNA. In CF patients, retention of viscous purulent secretions in the airways contributes both to reduced pulmonary function and to exacerbation of infection. These pulmonary secre- tions contain very high concentrations of extracellular DNA released by degenerating leukocytes. Dornase alfa is delivered to CF patients by inhalation of an aerosol mist produced by a pneumatic nebulizer; it hydrolyses the DNA in airway secretions and reduces their viscosity.

A significant disadvantage of inhalation therapy is the relatively short duration of drug action demanding multiple daily inhalation maneuvers, ranging up to 9 times a day [9].

Strategies for further advancements of inhalation therapy include the development of aerosolizable carrier systems with the aim to improve the drug effect, as well as patient's convenience and compliance. Today, researchers have made great strides in the development of pulmonary delivery technologies, both in terms of inhaler design and progresses in nanoscale carrier engineering. Today there are three main different classes of devices for pulmonary drug delivery: nebulizers, Metered Dose Inhalers (MDIs), and Dry Powder Inhalers (DPIs). These inhalers are based on different delivery mechanisms, and require different types of drug formulations. Furthermore, the development of new bioactive compounds such as nucleic acids and proteins, require the design of innovative delivery technologies.

Among the various drug delivery systems considered for pulmonary application, nanocarriers demonstrate several advantages for the treatment of respiratory diseases, for example prolonged drug release and cell-specific targeted drug delivery.

The development of an innovative nanocarrier, able to deliver the drug to the desired site of action, is highly dependent on the nature of the active substance and on its desired site and mode of action (Figure 1).

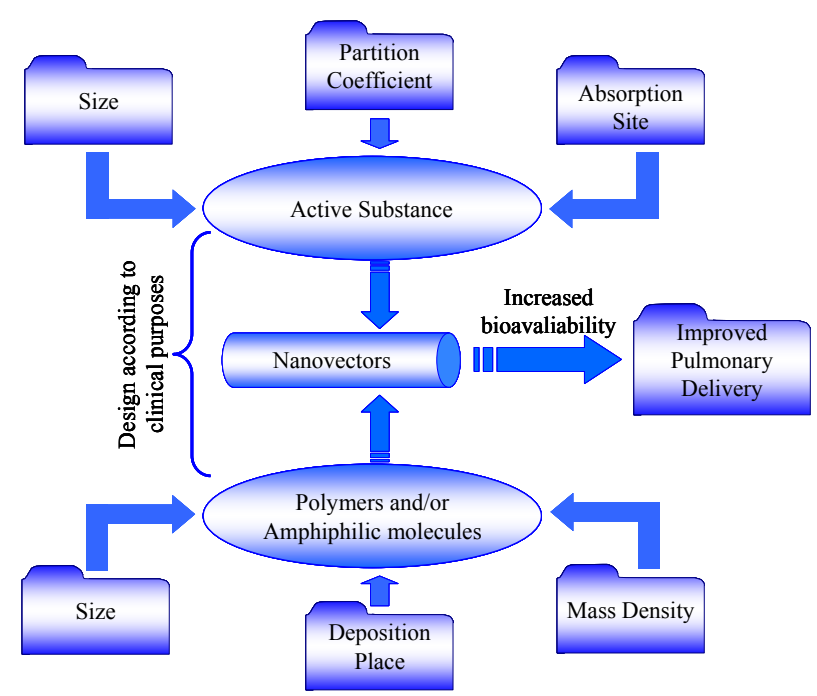

Figure 1. Factors affecting pulmonary delivery. 


\section{Practical Address in Pulmonary Delivery}

The development of drug delivery systems for pulmonary application requires a detailed knowledge of the lung in its healthy, as well as various diseased states. The lung consists of two functional parts, the airways (trachea, bronchi, and bronchioles) and the alveoli (gas exchange areas). The primary functions of the lungs are to enable gas exchange between the blood and the external environment, and to maintain homeostatic systemic $\mathrm{pH}$. The respiratory system is composed of the trachea, which bifurcates into the bronchi. The bronchi continue to branch into smaller bronchioles, and ultimately the terminal bronchi, which end with the alveolar sacs.

Gas exchange between airspaces and blood capillaries occurs in the respiratory region, which includes the respiratory bronchioles, the alveolar ducts and the alveolar sacs.

The surface area of the alveolar epithelium reaches $100 \mathrm{~m}^{2}$, which is enormous as compared to the $0.25 \mathrm{~m}^{2}$ surface area of the airways [10,11]. The alveoli have a thin, single cell layer. The distance from the air in the alveolar lumen to the capillary blood flow is less than $400 \mathrm{~nm}$. The large surface area of the alveoli and the intimate air-blood contact in this region make the alveoli less well protected against inhaled substances, such as nanoparticles, as compared to the airways [12]. Despite the numerous advantages above reported, pulmonary administration of drugs shows, at the same time, several problems related, for instance, to the various clearance mechanisms and the mucous layer. Mucociliary clearance is one of the most important defence mechanisms to eliminate dust and microorganisms in the lungs [13]. The mucus is produced by goblet cells and sub-mucosa glands. It covers the entire airway surface and its thickness ranges from $5 \mu \mathrm{m}$ to $55 \mu \mathrm{m}[14,15]$. It consists of an upper gel phase made of $95 \%$ water, $2 \%$ mucin, a highly glycosylated and entangled polymer, as well as salts, proteins and lipids [16]. A periciliary liquid layer underlies the mucus gel and its low viscosity allows effective cilia beating. The coordinated, rhythmic beating of the cilia constantly moves this mucous layer toward the proximal airways, where it is either swallowed or expectorated (mucociliary clearance). Particles settling in the peripheral lung have been reported to have a residence time of approximately $24 \mathrm{hr}$ in a healthy adult patient.

Pulmonary surfactants are responsible for biophysical stabilizing activities and innate defence mechanisms. They line the alveolar epithelial surfaces and overflow into the conductive airways so that the surfactant film is continuous between alveoli and central airways [17]. Pulmonary surfactants are composed of phospholipids $(80 \%$, half of which being dipalmitoylphosphatidylcho- line), neutral lipids ( $5 \%-10 \%$, mainly cholesterol), specific surfactant proteins $(5 \%-6 \%)$ and non-specific proteins $(3 \%-4 \%)[18]$.

Phospholipids are mainly responsible for the formation of the surface active film at the respiratory air-liquid interface. In water, phospholipids are self-organized in the form of bilayers. Bilayers are also the structural form in which surfactants are assembled and stored by pneumocytes in lamellar bodies.

Specific surfactant proteins include SP-A, SP-B, SP-C and SP-D. SP-A and SP-D are hydrophilic while SP-B and SP-C are hydrophobic. SP-A is able to bind multiple ligands, including sugars, $\mathrm{Ca}^{2+}$ and phospholipids. This property allows SP-A to bind to the surface of pathogens, contributing to their elimination from the airways. Recognition of SP-A by specific receptors on alveolar macrophages stimulates phagocytosis of the pathogens. SP-B is strictly required for the biogenesis of pulmonary surfactants and their packing into lamellar bodies. Both, SP-B and SP-C promote the rapid transfer of phospholipids from bilayers stores into air-liquid interfaces.

Luminal airway and alveolar macrophages are at the forefront of lung defence and their primary role is to participate in innate immune responses, such as, chemotaxis, phagocytosis, and microbial killing [19]. They also downregulate adaptive immune responses and protect the lung from T-cell-mediated inflammation [20]. Macrophages are tightly applied on the surface of respiratory epithelia. They are immersed in the lung lining fluid beneath the surfactant film.

The lung presents a remarkably lower level of metabolism than the gastrointestinal tract and liver. Yet, various peptidases are distributed on the surface of different cell types in the lung, including bronchial and alveolar epithelial cells, submucosal glands, smooth muscles, endothelial cells and connective tissue.

Proteases play an essential role in cell and tissue growth, differentiation, repair, remodelling, cell migration and peptide-mediated inflammation [21]. Proteases can also be released in the airspaces by activated macrophages and neutrophils in case of inflammatory reactions in the respiratory tract [22]. Blood supply to the lungs is divided among the pulmonary and systemic circulations. The pulmonary circulation consists of the pulmonary artery that leaves the right heart, branches into a dense pulmonary capillary bed that surrounds the alveoli and finally coalesces into the pulmonary vein that drains into the left heart. One hundred percent of the cardiac output flows through the pulmonary circulation. Its principal functions are gas exchange with air in the alveoli and nutrients supply to terminal respiratory units. The lungs receive a second blood supply via the systemic circulation, commonly referred to as the bronchial circulation. 
The bronchial circulation originates from the aorta and provides oxygenated blood and nutrients to all structures of the tracheobronchial tree. Lymphatic vessels exist in close proximity of major blood vessels and of the airways [23].

This high level of vascularization, and the large surface area combined with an extremely thin barrier between the pulmonary lumen and the capillaries, create conditions that are well suited for efficient mass transfer [24].

Particle deposition in the lungs occurs by inertial impaction, sedimentation. In order to evaluate the capacity of inhaled particles to reach different regions of the respiratory tract, the so-called aerodynamic diameter of such particles must be considered. The aerodynamic diameter of a particle, $d_{a e r}$, is equivalent to the diameter of a unit density $\left(\rho_{0}\right)$ sphere that has the same terminal velocity in still air as the particle:

$$
d_{\text {aer }}=d \sqrt{\frac{\rho}{\rho_{0} X}}
$$

where $d$ is the geometric diameter of the particle, $\rho$ is the particle density and $X$ is the particle dynamic shape factor denoting deviation of shape from sphericity [25].

Actually, particles larger than $5 \mu \mathrm{m}$ in diameter are generally subject to inertial impaction in the oropharyngeal region, or sedimentation in the bronchial region, where delivered drug may be expected to have little systemic therapeutic effect. At the other extreme, particles with diameters substantially smaller than $1 \mu \mathrm{m}$, although capable to reach the alveolar region, they are not capable to deposit and are thus exhaled. Particles with aerodynamic diameters between 1 and $5 \mu \mathrm{m}$ are expected to bypass deposition in the mouth and throat and efficiently deposit in the lung periphery [26].

\section{Innovative Approaches in Lung Delivery}

The successful integration of novel drugs with devices capable of delivering defined doses to the respiratory tract has resulted in a proven track record for inhalation as a route of administration that limits systemic exposure and provides localized topical delivery. Thus, a number of orally inhaled products have been successfully developed over the last 50 years, providing symptomatic relief to millions of patients with asthma and chronic obstructive pulmonary disease (COPD).

Nowadays, biopharmaceuticals and conventional drugs are frequently engineered or incorporated in carriers in order to direct their fate in preferential pathways $[27,28]$. Nano-size drug carriers can incorporate various therapeutics (e.g., poorly water soluble drugs) and present several advantages for drug delivery to the lung include- ing controlled release, protection from metabolism and degradation, decreased drug toxicity and targeting capabilities.

Nanotechnology is the engineering and manufacturing of materials at the atomic and molecular scale. In its strictest definition from the National Nanotechnology Initiative, nanotechnology (NIH Roadmap Initiatives, http://nihroadmap.nih.gov/initiatives.asp.), it refers to structures roughly in the $1-100 \mathrm{~nm}$ size regime in at least one dimension. Despite this size restriction, nanotechnology commonly refers to structures that are up to several hundred nanometers in size and that are developed by topdown or bottom-up engineering of individual components [29]. Bioactive delivery nanosystems (nanocarriers) in general, and drug delivery in particular, constitute a significant domain of nanomedicine.

Nanomedicine can be defined as the application of nanotechnology to medicine. Artificial nanostructures are of the same size as biological entities and can readily interact with biomolecules on both the cell surface and within the cell (Figure 2).

The understanding of the fate of nanomedicines in the lungs is important because fate and therapeutic activity are closely related. Interaction of nanomedicines with cells of the respiratory system will determine the pharmacodynamic response. For instance, the rapid uptake of particles by alveolar macrophages can be a way of targeting anti-tuberculosis drugs to this type of cells [30]. Conversely, macrophages uptake represents a clearance pathway for drugs acting on other cells within the lungs (e.g., $\beta 2$ mimetics) [31].

There are numerous types of nanoparticle systems now being explored for drug delivery to lungs, especially in cancer treatment [32]. The types of nanovectors used at present in research for cancer therapeutic applications include polymeric nanoparticles, protein nanoparticles, ceramic nanoparticles, viral nanoparticles and metallic nanoparticles [33].

A further innovation in the treatment of lung cancer concerns the development of inhalable nanoparticles (NPs) to obtain cytotoxicity mediated by alveolar macrophages [34,35].

Lipid-based carrier systems, including liposomes and their nanoversions (nanoliposomes), are among the most promising encapsulation technologies employed in the rapidly developing field of nanotechnology. Liposomes are the most extensively investigated system for controlled drug delivery to the lungs [36]. A few lipo-someencapsulated antibiotics have been delivered to the lungs in phase II clinical trials. These include amikacin [37] and ciprofloxacin [38]. Multiple treatment cycles with ARIKACETM (liposomal amikacin for inhalation) showed sustained improvement in lung function with significant 


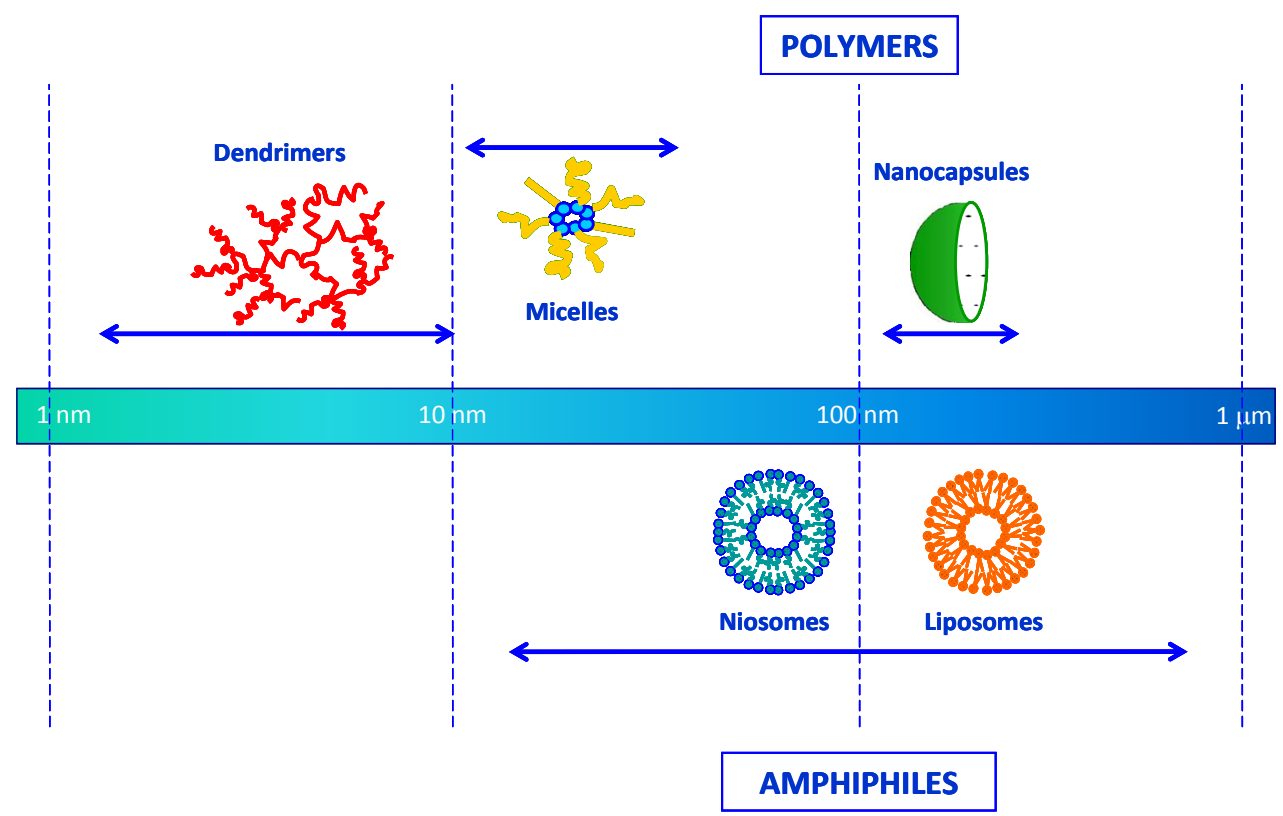

Figure 2. Types of nanosize systems used for drug delivery and targeting. The blue axsis represents the diameters of nanovectors.

reduction in bacterial density in CF patients who have chronic Pseudomonas lung infections [39].

A nanoscale liposomal formulation of amikacin has been shown to slowly release the drug in rat lungs and to penetrate Pseudomonas biofilms and CF sputum in vitro [40].

Mitsopoulos and Suntres [41] reported that the delivery of $\mathrm{N}$-acetylcysteine as a liposomal formulation improves its effectiveness in counteracting Paraquat-induced cytotoxicity.

Liposomal drug dry powder formulations, realized to obtain novel devices capable of delivering defined doses of drugs, represent promising tools for pulmonary drug administration, such as selective localization of drug, reduced local and systemic toxicities, increased patient compliance and high dose loading.

In liposomal dry powder formulations, drug encapsulating liposomes are homogenized, dispersed into the carrier and converted into dry powder by using freeze drying, spray drying or supercritical fluid technologies.

Huang et al. developed a formulation of liposomal salbutamol sulfate (SBS) with high encapsulation efficiency (more than $80 \%$ ) formulated in a dry powder inhaler (DPI) for the treatment of asthma, offering the promising possibility of localized pulmonary liposomal SBS delivery in the anhydrous state [42].

The most commonly used liposomes are composed of lung surfactants and synthetic lipids. Liposomal formulation have been proposed to delivery anticancer drugs, corticosteroids, immunosuppressants, antimicotic drugs, antibiotics for local pulmonary infections and $\mathrm{CF}$ and opioid analgesics for pain management. Many of them have reached the stage of clinical trials for the treatment of several pulmonary diseases [43].

Surfactant vesicles are analogous to liposomes but have several advantages over them and were proposed for pulmonary delivery. A novel approach was developed for the preparation of controlled release proniosomederived niosomes, using sucrose stearates as non-ionic biocompatible surfactants for the nebulisable delivery of cromolyn sodium [44]. An attempt has been made to incorporate anti-tuberculosis drugs (ATD's) in the prepared niosomes. High encapsulation efficiency was obtained and should an advantage to solve the problem of multi-drug resistance in case of tuberculosis [45].

Various nonionic surfactants of sorbitan ester class together with cholesterol were proposed to prepare niosomes containing rifampicin [46]; furthermore, Polysorbate 20 vesicles were studied in pulmonary glucocorticoid delivery $[47,48]$.

A new trend in vesicles lung delivery is addressed to obtain efficient and safe vaccine delivery systems. A liposomal vaccine (MLB) based on xenogeneic human Basic fibroblast growth factor and monophosphoryl lipid A (MPLA) was proposed to induce humoral immunity through cross-reaction, to mediate Th1 immune response preferentially and to enhance antitumor activity in vivo. [49].

A promising application of nanocarriers to lung targeting is related to gene delivery. Gene therapy is cur- 
rently being developed for a wide range of acute and chronic lung diseases, including $\mathrm{CF}$, cancer and asthma. Several authors [50-52] developed a highly efficient nanocomposite aerosol for pulmonary gene delivery, consisting of a biodegradable polymer core.

Serious respiratory diseases, due to their lethality and prevalence, have attracted particular attention as targets of small interfering RNA (siRNA) - mediated therapeutic agents, also because of lung's accessibility leading to both local and systemic effects. However, one of the major challenges to achieve the siRNA therapeutic potential in lung diseases is to deliver the siRNAs to the lung tissue, in particular, to the target cells with high efficiency and high specificity [53].

Several clinical trials have been conducted in order to assess the efficacy and safety of pulmonary DNA delivery using viral and non-viral vectors, especially in the case of CF. Yet, none of these formulations have been pursued due to low transfection efficiency, transient gene expression or immune elimination of the gene vector. Identification of the barriers to cell transfection might help to improve gene transfer efficiency of non-viral vectors [51]. An efficient and safe cationic lipid, 6lauroxyhexyl lysinate (LHLN), was proposed to prepare cationic liposomes. In vitro tests showed that, compared with Lipofectamine2000, the new cationic liposome formulation using LHLN exhibited lower cytotoxicity and similar transfection efficiency in A549 and HepG2 lung cancer cells [54].

Ishitsuka et al. developed a multifunctional envelopetype nano device (MEND), in which plasmid DNA is condensed using a polycation to form a core particle that is encapsulated in a lipid envelope, modified with the IRQ peptide (IRQRRRR) to enhance transgene expression in lungs [55].

Clinical applications of liposomes and nanoparticles for drug delivery to the respiratory tract are still in early stages. The key to future innovation may lie at the interface between biology and particle engineering. Improved understanding of biological processes including particle clearance, cellular targeting, intracellular trafficking, and drug absorption are needed to better design formulations that deliver to the "target" with the optimal balance of pharmacodynamic, pharmacokinetic, and safety profiles. More specifically, continued advances are needed in the development of: 1) controlled release formulations; 2) formulations with improved regional targeting within the lungs (e.g., airway versus alveoli and vice versa); 3) formulations containing active targeting moieties; 4) formulation strategies for improving the systemic bioavailability of inhaled macromolecules; 5) formulation strategies for delivering macromolecules, including siRNA and DNA into cells; and 6) formulations with improved dose consistency. It is likely that such innovation will require the development of novel excipients and particle engineering strategies. Future innovation must also take into account the changing marketplace and the diverse set of customers (patient, healthcare professional, heath authorities, payers, and politicians) who must be satisfied. The pharmacoeconomics of new delivery systems will be closely scrutinized, so it is imperative that cost factors should be taken into account. Otherwise, the new technology option may overshoot the evolving inhalation marketplace.

\section{Toxicity of Nanoparticles to the Lung}

Epidemiological studies have confirmed a positive correlation between levels of particulate pollution and increased morbidity and mortality rates among general populations [56].

The adverse health effects seem to be dominated by pulmonary symptoms. For instance, many reports have addressed that occupational exposure of inhaled rigid nanoparticles (NPs) can lead to respiratory diseases such as pneumoconiosis (pulmonary fibrosis) and bronchitis $[57,58]$.

Increasing inhalation of ambient ultrafine particles has been linked with exacerbation of respiratory symptoms and mortality among COPD sufferers [59]. It has also been documented that NPs can instigate oxidative stress and cellular toxicity in various types of cells [60].

It was also reported that chronic exposure to NPs can potentially predispose humans to lung inflammation and increase the risk of COPD.

A concentration range of NPs within the level found in ambience and in nanotechnology industries [61] can promote mucin aggregation.

The second safety aspect of deep lung deposition is the interaction of nanoparticles with the alveolar environment that is covered with a thin surfactant film. This film, as above reported, has important physiological functions such as the acceleration of gas exchange and the lowering of the surface tension in the alveolar space. Such functions may be compromised by inhalable nanoparticles that may cause life threatening consequences. Therefore, the compatibility of a delivery system with the alveolar environment must be always carefully considered $[62,63]$.

For these reasons vesicular nanocarriers, composed of lung surfactants and/or synthetic amphiphiles may provide an efficient delivery system for the treatment of pulmonary disorders due to their biocompatibility, biodegradability and non-toxic nature [64].

\section{Conclusions}

Despite the many promising proof of concepts of various 
delivery technologies, there is still a long way ahead that must be covered. This means there are still many challenges that are being faced, which, in turn, mean there are many chances for the academic and industrial scientist to improve formulations and make a decisive impact.

Further research efforts are needed to ensure the safety of long-term in vivo applications and the development of scale up from laboratory to industry in order to reach, within a few years, the safety and large-scale production at affordable costs of innovative lung delivery technologies.

\section{REFERENCES}

[1] W. Yang, J. I. Peters and R. O. Williams III, "Inhaled Nanoparticles-A Current Review," International Journal of Pharmaceutics, Vol. 356, No. 1-2, 2008, pp. 239247. doi:10.1016/j.ijpharm.2008.02.011

[2] J. S. Patton and P. R. Byron, "Inhaling Medicines: Delivering Drugs to the Body through the Lungs," Nature Reviews Drug Discovery, Vol. 6, No. 1, 2007, pp. 67-74. doi: $10.1038 / \operatorname{nrd} 2153$

[3] E. Marttin, J. C. Verhoef, S. G. Romeijin and F. W. H. M. Merkus, "Effects of Absorption Enhancers on Rat Nasal Epithelium in Vivo: Release of Marker Compounds in the Nasal Cavity," Pharmaceutical Research, Vol. 12, No. 8, 1995, pp. 1151-1157. doi:10.1023/A:1016207809199

[4] F. W. H. M. Merkus, N. G. M. Schiepper, W. A. J. J. Hermens, V. S. G. Romeijin and J. C. Verhoef, "Absorption Enhancers in Nasal Drug Delivery: Efficacy and Safety," Journal of Controlled Release, Vol. 24, No. 1-3, 1993, pp. 201-208. doi:10.1016/0168-3659(93)90179-9

[5] W. T. Cefalu, "Concept, Strategies, and Feasibility of Noninvasive Insulin Delivery," Diabetes Care, Vol. 27, No. 1, 2004, pp. 239-246. doi:10.2337/diacare.27.1.239

[6] L. Illum, "Nasal Drug Delivery: New Developments and Strategies," Drug Discovery Today, Vol. 7, No. 23, 2002, pp. 1184-1189. doi:10.1016/S1359-6446(02)02529-1

[7] J. J. Neumiller and R. K Campbell, "Technosphere Insulin: An Inhaled Prandial Insulin Product," BioDrugs, Vol. 24, No. 3, 2010, pp. 165-172. doi:10.2165/11536700-000000000-00000

[8] A. Rossiter, C. P. Howard., N. Amin, D. J. Costello and A. H. Boss, "Technosphere ${ }^{\circledR}$ Insulin: Safety in Type 2 Diabetes Mellitus," ADA Scientific Sessions, Orlando Florida, June 2010.

[9] T. Gessler, W. Seeger and T. Schmehl, "Inhaled Prostanoids in the Therapy of Pulmonary Hypertension," Journal of Aerosol Medicine, Vol. 21, No. 1, 2008, pp. 1-12. doi:10.1089/jamp.2007.0657

[10] J. D. Crapo, B. E. Barry, P. Gehr, M. Bachofen and E. R. Weibel, "Cell Number and Cell Characteristics of the Normal Human Lung," American Review of Respiratory Disease, Vol. 126, No. 3, 1982, pp. 332-337.

[11] R. R. Mercer, M. L. Russell, V. L. Roggli and J. D. Crapo, "Cell Number and Distribution in Human and Rat Air- ways," American Journal of Respiriratory Cell and Molecular Biology, Vol. 10, No. 6, 1994, pp. 613-624.

[12] H. M. Courrier, N. Butz and T. F. Vandamme, "Pulmonary Drug Delivery Systems: Recent Developments and Prospects," Critical Reviews in Therapeutic Drug Carrier Systems, Vol. 19, No. 4-5, 2002, pp. 425-498. doi:10.1615/CritRevTherDrugCarrierSyst.v19.i45.40

[13] C. P. Van der Schans, "Bronchial Mucus Transport," Respiratory Care, Vol. 52, No. 9, 2007, pp. 1150-1158.

[14] M. T. Clunes and R. C. Boucher, "Cystic Fibrosis: The Mechanisms of Pathogenesis of an Inherited Lung Disorder," Drug Discovery Today Disease Mechanism, Vol. 4, No. 2, 2007, pp. 63-72. doi:10.1016/j.ddmec.2007.09.001

[15] S. K. Lai, Y. Y. Wang and J. Hanes, "Mucus-Penetrating Nanoparticles for Drug and Gene Delivery to Mucosal Tissues," Advanced Drug Delivery Reviews, Vol. 61, No. 2, 2009, pp. 158-171. doi:10.1016/j.addr.2008.11.002

[16] R. Bansil and B. S. Turner, "Mucin Structure, Aggregation, Physiological Functions and Biomedical Applications," Current Opinion in Colloid \& Interface Science, Vol. 11, No. 2-3, 2006, pp. 164-170. doi: 10.1016/j.cocis.2005.11.001.

[17] W. Bernhard, P. L. Haslam and J. Floros, "From Birds to Humans: New Concepts on Airways Relative to Alveolar Surfactant," American Journal of Respiriratory Cell and Molecular Biology, Vol. 30, No. 1, 2004, pp. 6-11. doi:10.1165/rcmb.2003-0158TR

[18] J. Perez-Gil, "Structure of Pulmonary Surfactant Membranes and Films: The Role of Proteins and Lipid-Protein Interactions," Biochimica et Biophysica Acta (BBA)Biomembranes, Vol. 1778, No. 7-8, 2008, pp. 1676-1695. doi:10.1016/j.bbamem.2008.05.003

[19] M. Geiser, "Update on Macrophage Clearance of Inhaled Micro- and Nanoparticles," Journal of Aerosol Medicine and Pulmonary Drug Delivery, Vol. 23, No. 4, 2010, pp. 207-217. doi:10.1089/jamp.2009.0797

[20] P. G. Holt, D. H. Strickland, M. E. Wikstrom and F. L. Jahnsen, "Regulation of Immunological Homeostasis in the Respiratory Tract," Nature Reviews Immunology, Vol. 8, 2008, pp. 142-152. doi:10.1038/nri2236

[21] V. van der Velden and A. R. Hulsmann, "Peptidases: Structure, Function and Modulation of Peptide-Mediated Effects in the Human Lung," Clinical \& Experimental Allergy, Vol. 29, No. 4, 1999, pp. 445-456. doi:10.1046/j.1365-2222.1999.00462.x

[22] F. Buhling, D. Groneberg and T. Welte, "Proteases and Their Role in Chronic Inflammatory Lung Diseases," Current Drug Targets, Vol. 7, No. 6, 2006, pp. 751-759. doi: $10.2174 / 138945006777435362$

[23] S. El-Chemaly, G. Pacheco-Rodriguez, Y. Ikeda, D. Malide and J. Moss, "Lymphatics in Idiopathic Pulmonary Fibrosis: New Insights into an Old Disease," Lymphatic Research and Biology, Vol. 7, No. 4, 2009, pp. 197-203. doi:10.1089/1rb.2009.0014

[24] J. D. Brain, "Inhalation, Deposition, and Fate of Insulin and Other Therapeutic Proteins," Diabetes Technology \& 
Therapeutics, Vol. 9, Suppl. 1, 2007, p. S-4-15. doi:10.1089/dia.2007.0228

[25] W. C. Hinds, "Uniform Particle Motion," In: W. C. Hinds, Ed., Aerosol Technology: Properties, Behavior and Measurement of Airborne Particles, Second Edition, Wiley, New York, 1999, pp. 53-55.

[26] J. Heyder, J. Gebhart, G. Rudolf, C. F. Schiller and W. Stahlhofen, "Deposition of Particles in the Human Respiratory Tract in the Size Range $0.005-15 \mu \mathrm{m}$," Journal of Aerosol Science, Vol. 17, No. 5, 1985, pp. 811-625. doi:10.1016/0021-8502(86)90035-2

[27] S. R. Schmidt, "Fusion-Proteins as BiopharmaceuticalsApplications and Challenges," Current Opinion in Drug Discovery \& Development, Vol. 12, No. 2, 2009, pp. 284-295.

[28] F. M. Veronese and G. Pasut, "Pegylation, Successful Approach to Drug Delivery," Drug Discovery Today, Vol. 10, No. 21, 2005, pp.1451-1458. doi:10.1016/S1359-6446(05)03575-0

[29] O. C. Farokhzad and R. Langer, "Impact of Nanotechnology on Drug Delivery," Acs Nano, Vol. 3, No. 1, 2009, pp. 16-20. doi:10.1021/nn900002m

[30] N. Nimje, A. Agarwal, G. K. Saraogi, N. Lariya, G. Rai, H. Agrawal and G. P. Agrawai, "Mannosylated Nanoparticulate Carriers of Rifabutin for Alveolar Targeting," Journal of Drug Targeting, Vol. 17, No. 10, 2009, pp. 777-787. doi:10.3109/10611860903115308

[31] J. Todoroff and R. Vanbever, "Fate of Nanomedicines in the Lungs," Current Opinion in Colloid \& Interface Science, Vol. 16, 2011, pp. 246-254. doi:10.1016/j.cocis.2011.03.001

[32] B. Haley and E. Frenkel, "Nanoparticles for Drug Delivery in Cancer Treatment," Urologic Oncology: Seminars and Original Investigations, Vol. 26, No. 1, 2008, pp. 57-64. doi:10.1016/j.urolonc.2007.03.015

[33] R. Singh and N. H. Singh, "Medical Applications of Nanoparticles in Biological Imaging, Cell Labeling, Antimicrobial Agents, and Anticancer Nanodrugs," Journal of Biomedical Nanotechnology, Vol. 7, No. 4, 2011, pp. 489-503. doi:10.1166/jbn.2011.1324

[34] K. M. Al-Hallak, S. Azarmi, A. Anwar-Mohamed, W. H. Roa and R. Löbenberg, "Secondary Cytotoxicity Mediated by Alveolar Macrophages: A Contribution to the Total Efficacy of Nanoparticles in Lung Cancer Therapy?" European Journal of Pharmaceutics and Biopharmaceutics, Vol. 76. No 1, 2010, pp. 112-119. doi:10.1016/j.ejpb.2010.05.002

[35] W. H. Roa, S. Azarmi, M. H. D. K. Al-Hallak, W. H. Finlay, A. M. Magliocco and R. Löbenberg, "Inhalable Nanoparticles, a Non-Invasive Approach to Treat Lung Cancer in a Mouse Model," Journal of Controlled Release, Vol. 150, No. 1, 2011, pp. 49-55. doi:10.1016/j.jconrel.2010.10.035

[36] H. M. Mansour, Y. S. Rhee and X. Wu, "Nanomedicine in Pulmonary Delivery," International Journal of Nanomedicine, Vol. 4, 2009, pp. 299-319. doi:10.2147/IJN.S4937
[37] J. Weers, B. Metzheiser, G. Taylor, S. Warren, P. Meers and W. R. Perkins, "A Gamma Scintigraphy Study to Investigate Lung Deposition and Clearance of Inhaled Amikacin-Loaded Liposomes in Healthy Male Volunteers," Journal of Aerosol Medicine and Pulmonary Drug Delivery, Vol. 22, No. 2, 2009, pp. 131-138. doi:10.1089/jamp.2008.0693

[38] P. Bruinenberg, D. Serisier, D. Cipolla and J. Blanchard, "Safety, Tolerability and Pharmacokinetics of Novel Liposomal Ciprofloxacin Formulations for Inhalation in Healthy Volunteers and Non-Cystic Bronchiectasis Patients," American Journal of Respiratory and Critical Care Medicine, B49 Meeting Abstract, American Thoracic Society 2010 International Conference, Vol. 181, Orleans, May 2010, p. A3192.

[39] O. O. Okusanya, S. M. Bhavnani, J. Hammel, P. Minic, L. J. Dupont, A. Forrest, G. J. Mulder, C. Mackinson, P. G. Ambroseand and R. Gupta, "Pharmacokinetic and Pharmacodynamic Evaluation of Liposomal Amikacin for Inhalation in Cystic Fibrosis Patients with Chronic Pseudomonal Infection," Antimicrobial Agents and Chemotherapy, Vol. 53, No. 9, 2009, pp. 3847-3854 doi:10.1128/AAC.00872-08

[40] P. Meers, M. Neville, V. Malinin, A. W. Scotto, G. Sardaryan, R. Kurumunda, C. Mackinson, G. James, S. Fisher and W. R. Perkins, "Biofilm Penetration, Triggered Release and in Vivo Activity of Inhaled Liposomal Amikacin in Chronic Pseudomonas Aeruginosa Lung Infections," Journal of Antimicrobial Chemotherapy, Vol. 61, No. 4, 2008, pp. 859-868. doi:10.1093/jac/dkn059

[41] P. Mitsopoulos and Z. E. Suntres, "Protective Effects of Liposomal N-Acetylcysteine against Paraquat-Induced Cytotoxicity and Gene Expression," Journal of Toxicology, Vol. 2011, 2011, Article ID 808967 (14 Pages). doi: $10.1155 / 2011 / 808967$

[42] W. H. Huang, Z. J. Yang, H. Wu , Y. F. Wong, Z. Z. Zhao and L. Liu, "Development of Liposomal Salbutamol Sulfate Dry Powder Inhaler Formulation," Biological \& Pharmaceutical Bulletin, Vol. 33, No. 3, 2010, pp. 512517. doi:10.1248/bpb.33.512

[43] A. Misra, K. Jinturkar, D. Patel, J. Lalani and M. Chougule, "Recent Advances in Liposomal Dry Powder Formulations: Preparation and Evaluation," Expert Opinion on Drug Delivery, Vol. 6, No. 1, 2009, pp. 71-89. doi: $10.1517 / 17425240802652309$.

[44] A. Abd-Elbary, H. M. El-laithy and M. I. Tadros, "Sucrose Stearate-Based Proniosome-Derived Niosomes for the Nebulisable Delivery of Cromolyn Sodium," International Journal of Pharmaceutics, Vol. 357, No. 1-2, 2008, pp. 189-198. doi:10.1016/j.ijpharm.2008.01.056

[45] K. M. Surinder, N. Jindal and G. Kaur, "Quantitative Investigation, Stability and in Vitro Release Studies of Anti-TB Drugs in Triton Niosomes," Colloids and Surfaces B: Biointerfaces, Vol. 87, No. 1, 2011, pp. 173-179. doi:10.1016/j.colsurfb.2011.05.018

[46] V. S. Jatav, S. K Singh, P. Khatri, A. K. Sharma and R. Singh, "Formulation and In-Vitro Evaluation of Rifampicin-Loaded Niosomes," Journal of Chemical and 
Pharmaceutical Research, Vol. 3, No. 2, 2011, pp. 199203.

[47] C. Terzano, L. Allegra, F. Alhaique, C. Marianecci and M. Carafa, "Non-Phospholipid Vesicles for Pulmonary Glucocorticoid Delivery," European Journal of Pharmaceutics and Biopharmaceutics, Vol. 59, No. 1, 2005, pp. 57-62. doi:10.1016/j.ejpb.2004.06.010

[48] C. Marianecci, D. Paolino, C. Celia, M. Fresta, M. Carafa and F. Alhaique, "Non-Ionic Surfactant Vesicles in Pulmonary Glucocorticoid Delivery: Characterization and Interaction with Human Lung Fibroblasts," Journal of Controlled Release, Vol. 147, No. 1, 2010, 127-135. doi:10.1016/j.jconrel.2010.06.022

[49] Z. Zhong, X. Wei, B. Qi, W. Xiao, L. Yang, Y. Wei and L. Chen, "A Novel Liposomal Vaccine Improves Humoral Immunity and Prevents Tumor Pulmonary Metastasis in Mice," International Journal of Pharmaceutics, Vol. 399, No. 1-2, 2010, pp 156-162. doi:10.1016/j.ijpharm.2010.07.053

[50] J. K. Lam, W. Liang and H. K. Chan, "Pulmonary Delivery of Therapeutic siRNA," Advanced Drug Delivery Reviews, 2011, Article in Press. doi:10.1016/j.addr.2011.02.006

[51] U. Griesenbach and E. W. Alton, "Gene Transfer to the Lung: Lessons Learned from More Than 2 Decades of CF Gene Therapy," Advanced Drug Delivery Reviews, Vol. 61, No. 2, 2009, pp. 128-139. doi:10.1016/i.addr.2008.09.010

[52] J. Nguyen, R. Reul, T. Betz, E. Dayyoub, T. Schmehl, T. Gessler, U. Bakowsky, W. Seeger and T. Kissel, "Nanocomposites of Lung Surfactant and Biodegradable Cationic Nanoparticles Improve Transfection Efficiency to Lung Cells," Journal of Controlled Release, Vol. 140, No. 1, 2009, pp. 47-54. doi:10.1016/j.jconrel.2009.07.017.

[53] X. Yuan, S. Naguib and Z. Wu, "Recent Advances of siRNA Delivery by Nanoparticles," Expert Opinion on Drug Delivery, Vol. 8, No. 4, 2011, pp. 521-536. doi:10.1517/17425247.2011.559223

[54] P. Li, D. Liu, X. Sun, C. Liu, Y. Liu and N. Zhang, "A Novel Cationic Liposome Formulation for Efficient Gene Delivery via a Pulmonary Route," Nanotechnology, Vol. 22, No. 24, 2011, Article ID 245104. doi:10.1088/0957-4484/22/24/245104

[55] T. Ishitsuka, H. Akita and H. Harashima, "Functional
Improvement of an IRQ-PEG-MEND for Delivering Genes to the Lung," Journal of Controlled Release, Vol. 154, No. 1, pp. 77-83. doi:10.1016/j.jconrel.2011.05.012

[56] M. R. Gwinn and V. Vallyathan, "Nanoparticles: Health Effects-Pros and Cons," Environmental Health Perspectives, Vol. 114. No. 12, 2006, pp. 1818-1825. doi: 10.1289/ehp.8871

[57] J. D. Byrne and J. A. Baugh, "The Significance of Nanoparticles in Particle-Induced Pulmonary Fibrosis," McGill Journal of Medicine, Vol. 11, No. 1, 2008, pp. 43-50.

[58] O. Lkhasuren, K. Takahashi and L. Dash-Onolt, "Occupational Lung Diseases and the Mining Industry in Mongolia," International Journal of Occupational and Environmental Health, Vol. 13, No. 2, 2007, pp. 195-201.

[59] T. Xia, N. Li and A. E. Nel, "Potential Health Impact of Nanoparticles," Annual Review of Public Health, Vol. 30, 2009, pp. 137-150. doi:10.1146/annurev.publhealth.031308.100155

[60] C. C. Huang, R. S. Aronstam, D. R. Chen and Y. W. Huang, "Oxidative Stress, Calcium Homeostasis, and Altered Gene Expression in Human Lung Epithelial Cells Exposed to ZnO Nanoparticles," Toxicology in Vitro, Vol. 24, No. 1, 2010, pp. 45-55. doi:10.1016/j.tiv.2009.09.007

[61] S. J. Klaine, P. J. J. Alvarez, G. E. Batley, T. F. Fernandes and R. D. Handy, "Nanomaterials in the Environment: Behavior, Fate, Bioavailability, and Effects," Environmental Toxicology and Chemistry, Vol. 27, No. 9, 2008, pp. 1825-1851.doi:10.1897/08-090.1

[62] M. Beck-Broichsitter, C. Ruppert, T. Schmehl, A. Guenther, T. Betz, U. Bakowsky, W. Seeger, T. Kissel and T. Gessler, "Biophysical Investigation of Pulmonary Surfactant Surface Properties upon Contact with Polymeric Nanoparticles in Vitro," Nanomedicine: Nanotechnology, Biology and Medicine, Vol. 7, No. 3, 2011, pp. 341-350. doi:10.1016/j.nano.2010.10.007

[63] S. Azarmi, W. H. Roa and R. Löbenberg, "Targeted Delivery of Nanoparticles for the Treatment of lung Diseases," Advanced Drug Delivery Reviews, Vol. 60, No. 8, 2008, pp. 863-875. doi:10.1016/j.addr.2007.11.006

[64] G. Taylor and I. Kellaway, "Pulmonary Drug Delivery," In: A. Hillery, A. Lloyd and J. Swarbrick, Eds., Drug Delivery and Targeting, Taylor \& Francis, New York, 2001, pp. 269-300. 